\title{
STUDIES ON PROTON AND ION INTERACTIONS IN PLASMA-BASED COLLECTIVE ACCELERATORS
}

\author{
R. L. Williams, Department of Physics, Florida A. \& M. University, Tallahassee, FL 32307, USA
}

\section{Abstract}

The acceleration of protons, helium ions and gold ions by high phase velocity, longitudinal, electrostatic waves is studied. A one-dimensional, single-particle model is used to determine the minimum energy needed for trapping by the waves, and the maximum energy to which these particles can be accelerated in the waves. Comparisons are made between the limits of ion and electron acceleration.

\section{INTRODUCTION}

Charged particles can be accelerated to very high energies over very short distances by trapping them in relativistic plasma waves. The very large longitudinal electrostatic fields and relativistic phase velocities of plasma waves are responsible for the large accelerating gradients. Much progress has been made in accelerating electrons in the past decade, however recently attention has been turning toward the acceleration of protons[1] and other ions in these waves. In this paper we present results of applying a one-dimensional computational model to the study of the acceleration of protons, helium ions and gold ions by high phase velocity, electrostatic waves. We note that a brief study of proton energy gain in the beat wave accelerator appears in Ref. [2] and a study of energy gain and trapping threshold for proton acceleration in the counterstreaming electron-beam wake-field accelerator appears in Ref. [3]. Details of the one-dimensional model used here, and previously applied to electron acceleration, appear in Ref. [4].

\section{TRAPPING AND ACCELERATION}

The wave frame trapping condition is that the particle's kinetic energy must be less than the potential energy of the wave. From this condition we can obtain the minimum, $\gamma_{\text {min }}$, and maximum, $\gamma_{\max }$, lab frame energies of the particles trapped in the wave, which are as follows[4]:

$$
\gamma_{\min }=\gamma_{p} \gamma_{w}\left(1-\beta_{p} \sqrt{1-\frac{1}{\gamma_{w}^{2}}}\right)
$$

and

$$
\gamma_{\max }=\gamma_{p} \gamma_{w}\left(1+\beta_{p} \sqrt{1-\frac{1}{\gamma_{w}^{2}}}\right)
$$

where

$$
\gamma_{p}=\left(1-\frac{v_{p}^{2}}{c^{2}}\right)^{-1 / 2}=\text { Lorentz relativistic factor for the }
$$

wave, also called "gamma phase"

$$
\begin{aligned}
& v_{p}=\text { phase velocity of the wave } \\
& c=\text { speed of light } \\
& \gamma_{w}=\left(1+\frac{n_{1}}{n_{o}} \frac{m_{e}}{m_{i}} \Gamma \gamma_{p} \beta_{p}^{2}\right)=\text { Lorentz factor for the }
\end{aligned}
$$

particle in the wave frame

$$
\begin{aligned}
& \beta_{p}=\sqrt{1-\frac{1}{\gamma_{p}^{2}}} \\
& n_{o}=\text { background plasma electron density } \\
& n_{1}=\text { electron density of the plasma wave } \\
& m_{e}=\text { rest mass of the electron } \\
& m_{i}=\text { rest mass of the ion } \\
& \Gamma=\left(1+\cos \theta_{o}\right)=\text { phase factor, where } \theta_{o} \text { equals zero }
\end{aligned}
$$

when the particle starts at the bottom of the wave's potential, as in the case of these studies.

In this 1-D model there are no radial fields, so that focusing and defocusing are not considered. We also assume that the particle starts at the minimum of the electrostatic potential well in the wave frame, then moves backwards to the peak of the potential, then reverses direction and moves forward to the bottom of the potential, where it started, at which point its energy is maximum. If the paticle were to go forward past this point it would lose energy. If the particle were to go backwards past the peak of the potential, then it would not be trapped. If the particle were to start at the point in the wave where the potential is zero, then $\theta_{o}$ would be equal to $\pi / 2$ in the phase factor. In this study the helium and gold ions are singly ionized. In the plots shown below the particle's energy and the wave's phase velocity are represented by the corresponding Lorentz relativistic factor, $\gamma$.

In Fig. 1 we plot the particle's energy versus gamma phase for the three ions considered. Shown are the maximum energy attained in the wave (the upper curves having increasing slopes) and the threshold energy needed for trapping (the lower curves having decreasing slopes). The maximum energy attained by an electron versus gamma phase (the straight line) is shown for comparison. In this figure, $n_{1} / n_{o}$ equals one. We see that very large injection energies and large phase velocities would be required for significant energy gain of these ions. Note also that the slopes of the ion maximum energy curves approach the slope of the electron maximum energy curve at high gamma phase. The gamma phase was plotted up to, arbitrarily, $10^{5}$. 
In Figs. 2, 3 and 4 are plotted the trapping threshold energies and the maximum energies for protons, helium ions and gold ions, respectively, versus $\varepsilon=n_{1} / n_{o}$ for the cases of gamma phase from 5 to 100,000 . In Fig. 5 is an expanded version of the proton curve showing details for the easiest of these cases, i.e. for protons at lower injection energies and smaller values of gamma phase. The trapping threshold and maximum energy curves for electrons are shown for comparison in Fig. 6.

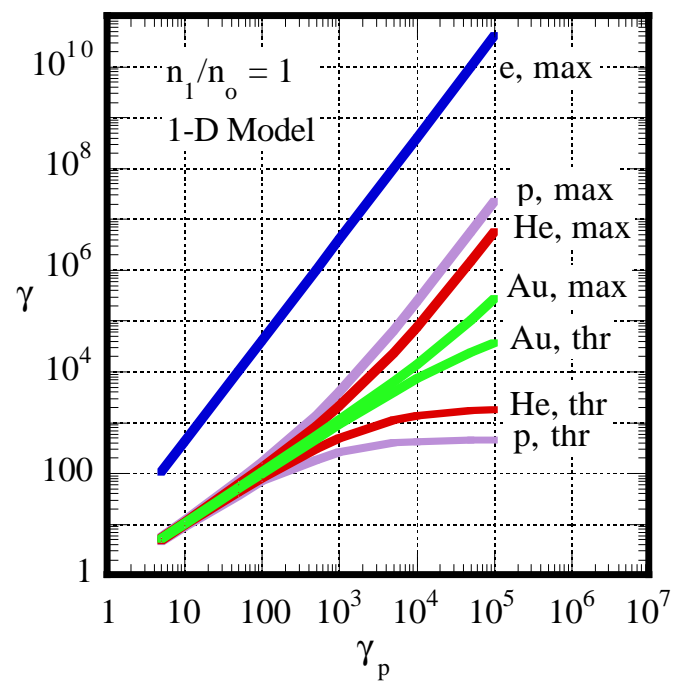

Figure 1: Trapping Threshold (thr) and maximum energy (max) curves for protons, helium ions and gold ions vs gamma phase; and the maximum energy curve for electrons.

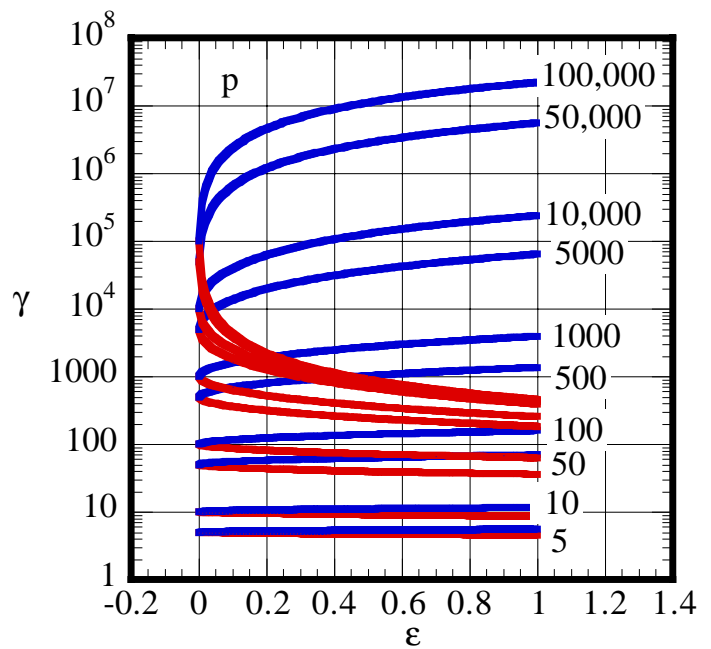

Figure 2: Proton trapping threshold and maximum energy versus $\varepsilon$ for gamma phase from 5 to 100,000 .

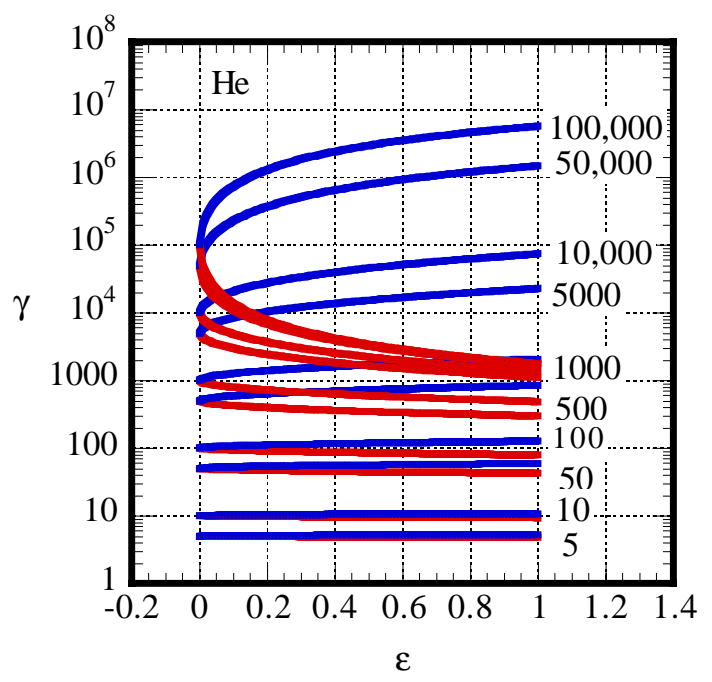

Figure 3: Helium trapping threshold and maximum energy versus $\varepsilon$ for gamma phase from 5 to 100,000 .

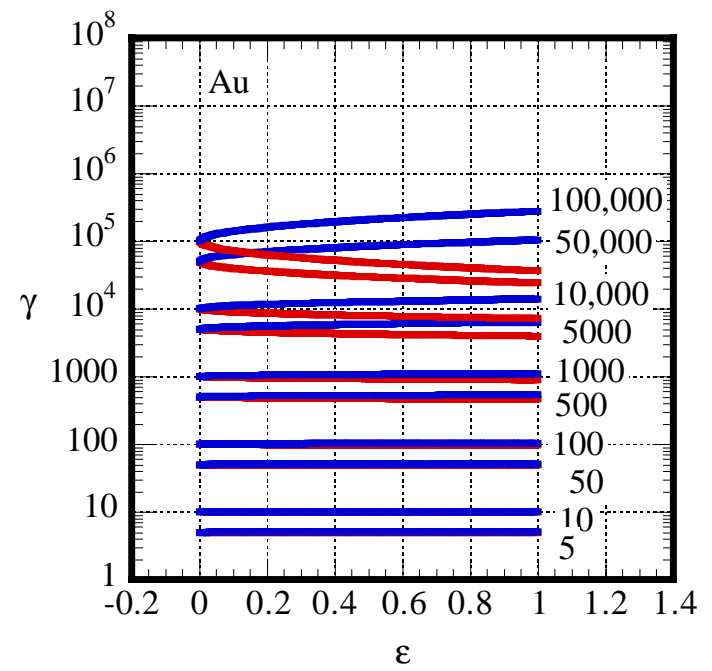

Figure 4: Gold trapping threshold and maximum energy versus $\varepsilon$ for gamma phase from 5 to 100,000 . 


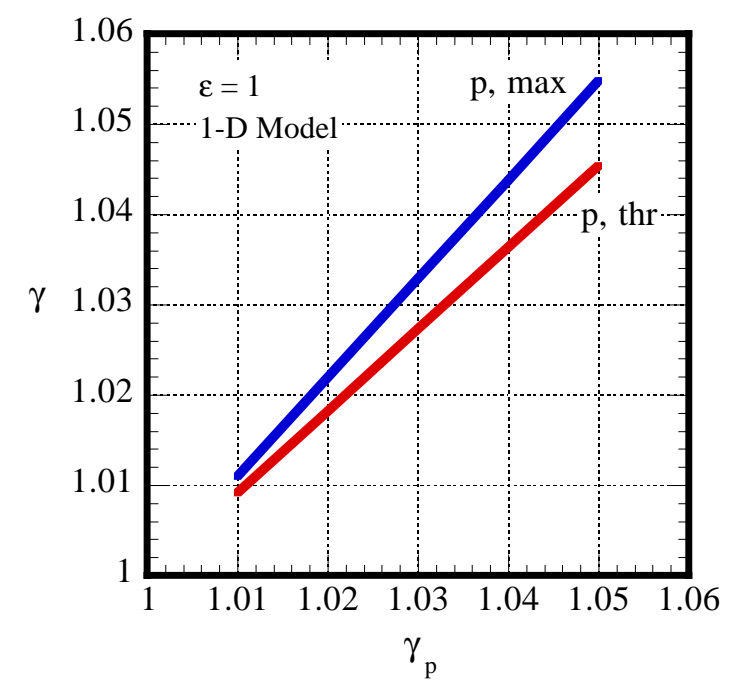

Figure 5: Expanded curves for proton trapping threshold (thr) and maximum energy (max) versus gamma phase from 1.01 to 1.05 for $\varepsilon=1$.

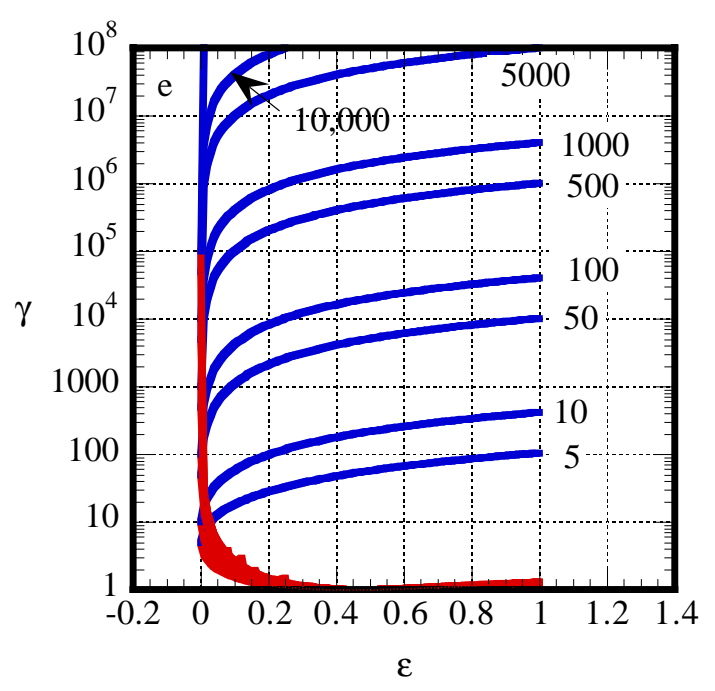

Figure 6: Electron trapping threshold and maximum energy versus $\varepsilon$ for gamma phase from 5 to 10,000 .

\section{CONCLUSIONS}

This study, using a 1-D single-particle model, has given some insight into the limits for high gradient acceleration in relativistic electrostatic waves for protons, and for two arbitrarily selected heavier ions, helium and gold. There are many factors that would modify these limits, such as two dimensional focusing/defocusing effects and various nonlinearities. Such waves might be able to boost the energy of these particles significantly after they have been accelerated to relativistic energies by conventional accelerators. Only small energy increases would be observed by injecting nonrelativistic particles into these plasma waves. In separate studies we are addressing acceleration lengths and two-dimensional effects.

\section{ACKNOWLEDGMENTS}

This work was supported by the Department of Energy under Grant No. DE-FG02-96ER40998.

\section{REFERENCES}

[1] A. Ogata and T. Katsouleas, Proceedings of the 1999 Particle Accelerator Conference, New York, 1999, p. 3713

[2] T. C. Katsouleas. Cross Magnetic Field Plasma Phenomena: Wave Studies, Particle Acceleration, Thermal Relaxation. Ph.D. Thesis, UCLA (1984), Appendix D.

[3] Y. T. Yan, Phys. Rev. A, 36(4), 1815 (1987).

[4] R. L. Williams, et al., Laser Part. Beams 8(3), 427 (1990). 